\title{
¿POR QUÉ NO TENEMOS TEORÍAS COHERENTES DE RELACIONES INTERNACIONALES SOBRE LA GLOBALIZACIÓN?
}

ArIe M. KaCowicz Mor Mitrani

\section{INTRODUCGIÓN}

LA GLOBALIZACIÓN SE HA CONVERTIDo en una de las palabras de moda del nuevo milenio tanto en el discurso académico como en el popular. Aunque las alusiones públicas a la globalización se han hecho más comunes a lo largo de las últimas dos décadas, hay vastos debates que siguen sin resolverse sobre su genealogía, trayectoria y aspectos distintivos. Lo que se ha hecho evidente es que nuestro propio tiempo de globalización política, económica y cultural desafía la separación estricta entre asuntos internos y exteriores, domésticos e internacionales, locales y globales. ${ }^{1}$ De aquí que la globalización ha puesto en duda las premisas ortodoxas y las concepciones de la teoría y de las prácticas de las relaciones internacionales, al menos en su variante de enfoque territorial convencional. Algunos inclusive cuestionan la identidad misma de las relaciones internacionales como un campo de investigación académica, ${ }^{2}$ argumentando que la globalización genera nuevos

${ }^{1}$ Ian Clark, "Beyond the Great Divide: Globalization and the Theory of International Relations”, Review of International Studies, vol. 24, núm. 4, 1998, pp. 479498; David Held y Anthony McGrew, "The Great Globalization Debate: An Introduction", en David Held y Anthony McGrew (eds.), The Global Transformation Reader: An Introduction to the Globalization Debate, Londres, Polity, 2003, pp. 1-50.

${ }^{2}$ Véase Colin Hay, "Theories of Globalization”, en Tim Dunne, M. Kurki y 
órdenes políticos y sociales que han remplazado a las "relaciones internacionales" como una descripción, no sólo de cómo se ve el mundo, sino de cómo debiéramos entender el papel de la política en el mundo.

Este artículo considera la cuestión de por qué la disciplina de las relaciones internacionales sale más bien perdiendo cuando se enfrenta con el concepto de "globalización" en el nivel teórico. Contra las realidades actuales de interdependencias intensificadas y de globalización, la teoría de las relaciones internacionales parece desconcertada, especialmente en lo que se refiere a su habilidad de incorporar el concepto teórico y las realidades de la globalización en sus programas convencionales de investigación. Con algunas excepciones que se explicarán a detalle en la segunda sección del artículo, los teóricos de las relaciones internacionales no se han ocupado directamente del concepto de "globalización", conque no hay teorías coherentes de la globalización dentro de las relaciones internacionales, sino más bien aproximaciones modestas que tratan algunas de sus facetas y dimensiones concretas. Este artículo discute este déficit teórico al proponer las siguientes preguntas: ¿cómo podríamos enfrentar la globalización desde la perspectiva de las relaciones internacionales?, ¿por qué no disponemos de teorías coherentes de relaciones internacionales sobre la globalización?, ¿qué debiéramos hacer al respecto?

Con base en la discusión sobre estas tres cuestiones, argumentamos que aunque hay una clara necesidad de teorizar la globalización en el marco de las teorías convencionales de las relaciones internacionales, existen serios impedimentos que nos limitan para desarrollar teorías coherentes en relaciones internacionales sobre la globalización, sobre todo a causa de la incompatibilidad inherente entre los aspectos de las teorías convencionales de relaciones internacionales y el concepto y las realidades de la globalización.

\footnotetext{
Steven Smith (eds.), International Relations Theories: Discipline and Diversity, Oxford, Oxford University Press, p. 2; James N. Rosenau, "Change, Complexity and Governance in Globalizing Space”, en J. Pierre (ed.), Debating Governance: Authority, Steering and Democracy, Oxford, Oxford University Press, 2000.
} 
Nuestro argumento principal se enfoca en la necesidad de destacar los nexos entre las relaciones internacionales y la globalización, según están encarnados por el papel de los Estados como agentes preponderantes del cambio en los procesos de la globalización. Esencialmente, en tanto que los Estados mantengan su rol de autoridades y mientras las relaciones interestatales perduren de manera predominante en la agenda de la política mundial, los avances teóricos, que siguen a la realidad empírica, deberían apuntar a dar cuentas del papel y la significación de la globalización desde una perspectiva interestatal (es decir, de relaciones inter-nacionales). Así, a diferencia de la discusión existente, sostenemos que las dimensiones global e internacional de la política global no son necesariamente contradictorias o mutuamente excluyentes, sino que están más bien interconectadas.

Las implicaciones políticas de los procesos de globalización pueden presentar nuevos problemas y desafíos políticos que provocan cambios y transformaciones en los patrones de los agentes políticos, las estructuras y las interacciones. Con todo, a final de cuentas, estos cambios y transformaciones tienen lugar bajo la autoridad de los Estados-nación, o al menos en interacción con sus ámbitos inter-estatales, de manera que los agentes principales que afrontan las implicaciones de la globalización siguen siendo los Estados, tanto individual como colectivamente según esquemas multilaterales. Argumentamos en este artículo que la política, especialmente la política interestatal, desempeña un papel crucial en nuestro esfuerzo por dar sentido a los efectos problemáticos de la globalización; también es parte vital del intento de controlar la globalización y de encontrar soluciones adecuadas a sus potenciales externalidades negativas. Así, los Estados, mediante sus gobiernos nacionales, actúan como "cinturones de transmisión", al mediar el impacto que tiene la globalización en las relaciones internacionales y las sociedades internas. ${ }^{3}$

Por lo tanto, sostenemos que el principal vínculo entre las relaciones internacionales y la teoría de la globalización depende de

${ }^{3}$ Arie M. Kacowicz, Globalization and the Distribution of Wealth: The Latin American Experience, 1982-2008, Cambridge University Press, 2013. 
nuevos campos empíricos de la política mundial, principalmente en los diferentes mecanismos de la gobernanza global y regional. Los mecanismos de gobernanza regional y global contemporáneos, a través de diversos foros multilaterales, han florecido en las últimas dos décadas en la expansión creciente de áreas temáticas que van más allá de asuntos "tradicionales" de la política entre Estados, incluidos temas como la seguridad humana, la disparidad económica, la ecológica y la de género, la salud y las pandemias globales.

Un ejemplo reciente relativamente exitoso de esa interacción se evidencia en la esfera económica, mediante estructuras y mecanismos de gobernanza económica global que se basan en la cooperación interestatal y las instituciones internacionales, que han lidiado relativamente bien con la crisis económica desde 2008. ${ }^{4}$ En el ámbito de la seguridad, a partir del fin de la Guerra Fría podemos identificar nuevos tipos de amenaza que crearon, respectivamente, nuevas justificaciones para la demanda de gobernanza de seguridad regional, especialmente debido a su naturaleza híbrida, interna y transnacional, la cual requiere mayor coordinación y participación de nuevos actores. Tales preocupaciones son claras en el hemisferio occidental en general (nuevos papeles para la OEA) y en América Latina en particular (UNASUR, CAN, CARICOM, MERCOSUR y CELAC), así como en la evolución de nuevos marcos de seguridad en el este de Asia (por ejemplo, el Grupo de Trabajo para Combatir el Terrorismo y el Crimen Transnacional del Foro Regional de la ASEAN). De manera similar, la nueva y revitalizada Unión Africana debe tratar con un amplio abanico de amenazas internas y transnacionales, incluidas la escasez de alimentos, la pandemia del VIH/sida, el tráfico de drogas, el terrorismo y el "mercenarismo" por parte de compañías militares privadas. ${ }^{5}$

Estas tendencias políticas ilustran el supuesto según el cual el proceso de globalización ha traído problemas (globales) en estas

${ }^{4}$ Daniel W. Drezner, "The Irony of Global Economic Governance: The System Worked”, Council on Foreign Relations International Institutions and Global Governance Program, Working Paper, 2012.

${ }^{5}$ Véase Arie M. Kacowicz y Galia Press-Bar-Nathan, "Regional Security Governance", en Tanja Boerjel y Thomas Risse (eds.), Oxford Handbook of Comparative Regionalism, Oxford, Oxford University Press, 2016. 
diferentes áreas temáticas en la medida en que desafían las capacidades de cualquier gobierno de resolverlos por sí solos, lo que reitera la necesidad de reconstruir las nociones básicas de política sobre una base multilateral. En este sentido, los Estados deben trabajar con otro tipo de actores si quieren responder adecuadamente a los retos de un mundo globalizado que cambia rápidamente. Sin embargo, esos mecanismos son tanto los resultados políticos de procesos de globalización como las principales esferas en las que los Estados dan forma y gobiernan a la política mundial; encarnan el vínculo esencial entre la globalización y las relaciones interestatales. Como se elaborará más adelante, entender la política internacional desde este ángulo requiere, primero, evaluar cómo debemos hacer frente a la globalización desde una perspectiva de Relaciones Internacionales, y cómo hacer teoría de la globalización, así como adoptar una agenda de investigación relevante dentro de la teoría de Relaciones Internacionales que refleje las transformaciones políticas y arroje luz sobre los papeles políticos que los Estados juegan en estos contextos.

\section{A. ¿Cómo debemos lidiar CON la "Globalización” DESDE UnA PERSPECTIVA DE RELACIONES INTERNACIONALES?}

El concepto de "globalización" se puso de moda en los años noventa y en el nuevo milenio, y rápidamente se volvió dominante en varias áreas académicas como la economía, la sociología y los estudios de medios de comunicación. Dado que en un primer momento se percibió la globalización como un fenómeno económico de movimientos transfronterizos, transnacionales, de bienes, personas y capital, se la exploró principalmente bajo la lente de la economía. El concepto pronto reemplazó al de "interdependencia", que había estado de moda en los años setenta y ochenta; se presenta como una fase intensificada de interdependencia que trasciende la autoridad y el control exclusivos de los Estados.

Uno de los rasgos principales que distinguen a la globalización de la interdependencia económica es el hecho de que la primera trasciende el mero ámbito económico, pues afecta a todos los ám- 
bitos sociales en distintas maneras y magnitudes, y se enfoca tanto en los procesos como en los resultados. La expansión de la idea de "globalización" y sus realidades a un rango amplio de ámbitos sociales hace que se mantenga como factor social; algunos incluso la consideran el orden social contemporáneo más importante y un proceso que determina y constituye las principales interacciones que componen nuestro mundo social. ${ }^{6}$

En efecto, varios campos de las ciencias sociales han desarrollado diversas formas de dar cuenta de la globalización. El paso del nivel internacional al nivel global de análisis supone una tentativa de diseño sistémico y complejo que da sentido a la visión de conjunto, en lugar de una visión compartimentada de las realidades sociales.

Hay una necesidad intrínseca de desarrollar nuevas teorías coherentes de relaciones internacionales sobre la globalización. Si, en efecto, estamos ante cambios ontológicos en el orden de la vida social, puede resultar imposible que las teorías tradicionales simplemente adopten esos cambios en sus premisas básicas. Al hablar de "cambios ontológicos", queremos decir que la globalización es más que un conjunto de cambios profundos en los flujos de capital y comercio; manifiesta un cambio sustancial de la manera en la que intentamos explicar el mundo y darle sentido. Así, hay académicos prominentes en el campo de los estudios de la globalización que llegan incluso a afirmar que el cambio sustantivo en el orden social que la globalización representa es tal que refleja una nueva revolución paradigmática, lo que conlleva establecer la globalización como un prisma teórico diferente para entender los problemas sociales contemporáneos. Por ejemplo, James Rosenau asevera que es necesario adoptar "nuevos conjuntos de perspectivas" en lo que respecta a la esencia de las relaciones humanas, los cuales puedan abarcar las condiciones nuevas y desconocidas de la globalización que desafían la división común entre los ámbitos internacional

${ }^{6}$ Véase Ulrich Beck, The Reinvention of Politics: Rethinking Modernity in the Global Social Order, Cambridge, Polity Press, 1997; James N. Rosenau y E. O. Czempiel (eds.), Governance without Government: Order and Change in World Politics, Cambridge, Cambridge University Press, 1992. 
e interno. Al mismo tiempo, los "viejos dinosaurios", los Estados, no sólo se las arreglaron para sobrevivir toda adversidad de todo tipo y reinventarse contra este cambio ontológico, sino que también respondieron adaptando nuevos papeles para lidiar con la globalización con mecanismos de gobernanza global y regional.

En muchos sentidos, el problema de la falta de teorías coherentes significa que nuestras teorías existentes con más frecuencia en este periodo de globalización nos resultan más insuficientes que antes, debido a los retos y las necesidades para la gobernanza regional y global. Así que si no obtenemos la visión de conjunto de una manera holística, nos arriesgamos al problema de elaborar teorías o modelos con muchas variables causales posibles que conducen a dificultades inherentes para aislar la importancia y el peso de cualquiera de las partes que componen el panorama general de la globalización.

Sin duda, el concepto de "globalización" es desafiante y desconcertante cuando se intenta hacer teoría sobre él, principalmente porque es un fenómeno mucho más englobante y complejo que las "relaciones internacionales" y, en consecuencia, es también mucho más ambiguo, menos preciso y, en cierta medida, incoherente incluso. Esto puede explicar por qué la discusión académica en relaciones internacionales y ciencia política en general tiende a enfocarse en el concepto de "política global" para denotar la globalización de las esferas políticas contemporáneas, más que la globalización misma.

Esta discusión sobre la "política global" se orienta a explicar y entender cómo y por qué procesos y dimensiones diferentes de globalización desafían y afectan formas políticas convencionales, a saber los Estados-nación como unidades políticas, así como el sistema internacional como una estructura política. Con frecuencia apunta a dos dimensiones dinámicas que desafían las distinciones tradicionales entre lo interno y lo internacional y entre lo territorial y lo no territorial. Primero, los procesos contemporáneos de globalización crean redes de poder superpuestas que atraviesan fronteras territoriales, lo que se denomina "formas policéntricas de gobernanza" o "redes de gobernanza" en nuestra jerga científica. Como tales, aumentan la presión sobre el modelo westfaliano 
de orden mundial basado en la norma de la soberanía y el territorio del Estado-nación. Segundo, ya no puede suponerse que el locus del poder político efectivo resida sencillamente en las manos de gobiernos nacionales soberanos, de manera que haya una difusión evidente del poder de los Estados hacia actores no estatales. ${ }^{7}$ Con todo, "la política global" tiende a ignorar los efectos importantes que ejerce la globalización sobre las prácticas e instituciones convencionales de las relaciones internacionales; concretamente, el papel de las interacciones y relaciones interestatales en la política mundial contemporánea. Dicho esto, varios académicos han hecho esfuerzos para establecer un vínculo teórico entre la teoría de relaciones internacionales y la globalización, con distintos grados de éxito, como se indicará en la siguiente sección del artículo.

\section{B. Teorizar sobre la globalización EN El MARCo DE LAS RELACIONES INTERNACIONALES}

La inserción de la "globalización" en los discursos públicos y académicos a principios de los años noventa llevó a amplios trabajos para definir los fenómenos, sondear sus características y evaluar sus posibles consecuencias. ${ }^{8}$ El principal debate teórico giraba en torno a la cuestión de saber si acaso y en qué medida podía provocar el declive del Estado-nación, la erosión de su soberanía y la desaparición del sistema westfaliano en su conjunto.

En un extremo de este debate podemos situar a los académicos que argumentan que no hay un fundamento claro para hacer teoría de la globalización en el marco de las relaciones internacionales, ya que la primera hace redundantes a estas últimas. El principal defensor de esta posición radical es James Rosenau, quien

${ }^{7}$ Véase por ejemplo Moises Naim, The End of Power: From Boardrooms to Battlefields and Churches to States, Why Being in Charge Isn't What Is Used to Be, Nueva York, Basic Books, 2013.

${ }^{8}$ James N. Rosenau, "Turbulence in World Politics: A Theory of Change and Continuity", en Richard W. Mansbach y Edward Rhodes (eds.), Global Politics in a Changing World: A Reader, Boston, Houghtin Miffin Co., 2003, pp. 21-22. 
anuncia una era de "política post-internacional". ${ }^{9}$ De acuerdo con él, el cambio turbulento en la política post-internacional se caracteriza por tres dinámicas de política mundial simultáneas, en el nivel micro de los individuos, en el nivel estructural del sistema internacional y en la interacción entre ellos, el nivel relacional micro-macro. El surgimiento de un sistema policéntrico y multifacético que incluye una infinidad de actores no estatales y sus efectos en las relaciones agente-estructura resulta en la necesidad de una nueva ontología para entender la política mundial. Esta nueva ontología, encarnada en el concepto de "gobernanza global", encierra la premisa de que el mundo actualmente se compone de esferas de autoridad que no son necesariamente consistentes con la división del espacio territorial del orden internacional tradicional de los Estados soberanos. ${ }^{10}$

La incompatibilidad entre la realidad contemporánea y el lente ontológico existente es también el centro del argumento de Jan Scholte de que teorías sociales y políticas tradicionales son inadecuadas para comprender integralmente las realidades de la globalización. Scholte sugiere una aproximación ecléctica y compleja que asocia los desarrollos globales en configuraciones geográficas con tendencias en producción, gobernanza, identidad y conocimiento. ${ }^{11}$ En un intento por identificar los grandes cambios en la realidad social conjuntamente con varias teorías sociales, concluye que sólo una síntesis de teorías sociales puede explicar las realidades sociales contemporáneas de un modo exhaustivo. ${ }^{12} \mathrm{Y}$ sin embargo, en vez de dar cuenta de la amplia gama de relaciones trans-planetarias, Scholte elige poner énfasis en el elemento de la supra-territorialidad, es decir en las tendencias de la conectividad

${ }^{9}$ Roseau, "Turbulence in World Politics", pp. 21-23.

${ }^{10}$ James N. Rosenau, “Toward and Ontology for Global Governance”, en Martin Hewson y Timothy J. Sinclair (eds.), Approaches to Global Governance Theory, Nueva York, suny Press, 1999, pp. 288-289, 294; y James N. Rosenau, "Governance in the $21^{\text {st }}$ Century", en Rorden Wilkinson (ed.), The Global Governance Reader, Londres, Routledge, 2005, pp. 45-46.

11 Jan Aart Scholte, Globalization: A Critical Introduction, 2a ed., Nueva York, Palgrave-Macmillan, 2005, p. 121.

${ }^{12}$ Scholte, Globalization: A Critical Introduction, p. 136. 
global que trascienden la geografía territorial y se desvinculan de ésta, conque se pasa por alto el hecho de que, si bien la territorialidad ha sido contenida y transformada, subsiste como un elemento importante que da sentido a la política mundial.

Los ensayos para teorizar los cambios en los modos tradicionales de relaciones internacionales no son nuevos. En la década de los setenta, Robert Keohane y Joseph Nye desarrollaron una opción "globalista" a las relaciones internacionales tradicionales mediante el modelo de la "interdependencia compleja" como alternativa al modelo realista de "seguridad nacional". Vislumbrando la globalización, definieron "globalismo" como "un estado del mundo que involucra redes de interdependencia a distancias multicontinentales". En esta definición, Keohane y Nye subrayaron lo que años más tarde se ha tendido a ignorar: que la globalización es el resultado de procesos políticos, en la medida en que la política interna y las instituciones políticas desempeñan un papel primordial en determinar cómo la globalización afecta las interacciones inter-nacionales (o sea, interestatales). En este sentido, la globalización no es una fuerza en sí misma, sino que más bien depende de varias condiciones políticas.

Esta afirmación, sin embargo, es menos evidente entre las explicaciones más contemporáneas de la globalización dentro de las relaciones internacionales; esas explicaciones tienden a presentar a la globalización más como una fuerza independiente que como una condición. Por ejemplo, Ian Clark, en uno de los estudios más sistemáticos sobre la globalización y las relaciones internacionales, plantea una relación mutuamente constitutiva entre la globalización y el Estado, dentro de la cual el cambio ocurre en la globalización en tanto estructura política, como en el Estado como el agente en la relación. ${ }^{13}$ Desde esta perspectiva, la globalización puede considerarse causa y efecto de las transformaciones que han tenido lugar en los órdenes internacional y mundial. De acuerdo con Clark, la globalización provoca una transformación en la naturaleza de los propios Estados, y como tal el orden internacional contemporáneo puede y debe describirse como un

13 Ian Clark, Globalization and International Relations Theory, Oxford, Oxford University Press, 1999, p. 5. 
orden de "Estados globalizados". ${ }^{14}$ No obstante, esta visión destaca esencialmente que los Estados son en cierta forma receptivos a las fuerzas de la globalización, las cuales puede decirse que han logrado cambiarlos y afectar el marco entero del orden internacional. Los efectos de la globalización en el orden internacional también están en el centro del estudio de Andrew Hurrell, que buscó analizar los cambios y las previsiones futuras de la sociedad internacional contemporánea en la era de la política global, refiriéndose a temas específicos como los derechos humanos y la globalización económica. ${ }^{15}$ Como Clark, Hurrell sitúa el proceso de globalización en el contexto de orden, pero se refiere a la existencia de un “orden global”, más que meramente a un orden internacional; lo hace poniendo énfasis en el desarrollo de una gobernanza global compleja en torno, pero también más allá, del Estado-nación.

Contrariamente a las perspectivas de la globalización de Clark y Hurrell que se centran en el Estado, en el otro extremo del espectro y dentro del contexto de una visión más crítica de la Escuela Inglesa, Barry Buzan sugiere un marco teórico alternativo para refinar la conceptualización de la globalización, con base en la idea de la "sociedad mundial". ${ }^{16}$ Esta idea se apoya en la interacción analítica entre los tres pilares de la Escuela inglesa: los conceptos de sistema internacional, sociedad internacional y sociedad mundial. Buzan concuerda con Clark en que no hay un simple juego de suma-cero entre la globalización y el sistema internacional estatal, y que ambos coexisten simultáneamente. Más específicamente, Buzan distingue entre la sociedad internacional y la mundial por su respectiva composición de actores: territoriales (Estados) y actores no territoriales (no estatales).

Mientras que estos tres ejemplos de estudios académicos constituyen intentos significativos para reunir los marcos teóricos de

${ }^{14}$ Ian Clark, "Globalization and the Post-Cold War Order", en Steve Smith y John Baylis (eds.), The Globalization of World Politics: An Introduction to International Relations, Oxford, Oxford University Press, 2001, pp. 639-646.

${ }^{15}$ Andrew Hurrell, On Global Order: Power, Values, and the Constitution of International Society, Oxford, Oxford University Press, 2007.

${ }^{16}$ Barry Buzan, From International to World Society? English School Theory and the Social Structure of Globalization, Cambridge, Cambridge University Press, 2004, p. 12. 
relaciones internacionales a la luz de la globalización, pareciera que muchas de estas explicaciones mezclan la globalización como fuente y la globalización como resultado. Más aún, más que comprender a la globalización o la política global desde una perspectiva de relaciones internacionales, usan a la globalización para explicar por qué y cómo la política mundial contemporánea se globaliza, pero no cómo la globalización afecta a las relaciones internacionales per se. Tampoco sorprende que como parte de su esfuerzo de introducir una nueva, y obviamente abstracta y compleja faceta a la teoría de relaciones internacionales, muchas de estas interpretaciones más que ofrecer teoría de relaciones internacionales clara y coherente sobre la globalización, ofrecen enfoques híbridos o marcos generales para el análisis.

La ausencia de una teoría coherente de relaciones internacionales y globalización puede explicar la visión aún más compleja que evolucionó en los años posteriores a la crisis económica global de 2008, en la cual muchos académicos dejaron de tratar de definir a la globalización en términos teóricos para evaluar y explicar sus consecuencias concretas, y cómo lidiar con ellas. En general, la discusión de los últimos años registra el paso de estudiar la globalización a comprender la necesidad de una gobernanza global compuesta de nuevos mecanismos políticos que trascienden al sistema estatal y son adecuadas para hacer frente a las implicaciones complejas de la globalización económica y otros problemas globales. Uno de los principales temas en este respecto es la noción de crisis múltiples propulsadas por la globalización y proyectadas en varios ámbitos de la realidad social contemporánea. ${ }^{17}$ Mientras que las discusiones sobre las crisis múltiples de la globalización tienden a adoptar puntos de vista críticos y normativos, ${ }^{18}$ que corresponden

17 En 2010, la revista Globalizations publicó un número especial editado por Barry K. Gills dedicado a las implicaciones políticas de la crisis económica y financiera. Véase Barry K. Gills, "The Return of Crisis in the Era of Globalization: One Crisis or Many?”, Globalizations, vol. 7, núms. 1-2, 2010, pp. 3-8.

${ }^{18}$ Véase, por ejemplo, James H. Mittelman, "Crisis and Global Governance: Money, Discourses, and Institutions”, Globalizations, vol. 7, núms. 1-2, 2010, pp. 157-172; Richard Falk, "A Radical World Order Challenge: Addressing Global Climate Change and the Threat of Nuclear Weapons", Globalizations, vol. 7, núms. 1-2, 2010, pp. 137-155. 
a la ola crítica actual de la teoría de la globalización, esas explicaciones académicas no pueden considerarse como teorías contemporáneas coherentes de relaciones internacionales. Más bien, se concentran en aspectos y dimensiones específicas de la globalización, ya que se tienden a distanciarse del ángulo interestatal (RI) y de la necesidad de elaborar teorías coherentes. Así pues, hacia el final de esta sección seguimos desconcertados por la interrogante que planteamos al inicio del artículo, sin respuesta clara todavía: ¿qué impide la formulación de teorías de relaciones internacionales coherentes sobre la globalización?

\section{Explicar el (relativo) vacío de teorías coherentes de RELACIONES INTERNACIONALES SOBRE LA GLOBALIZACIÓN}

Hasta ahora hemos hablado de la necesidad de adaptar las teorías de relaciones internacionales a los conceptos y las realidades de la globalización y mostrado algunos intentos que se han hecho. Sin embargo, esas explicaciones no necesariamente pueden y raramente aspiran a introducir y elaborar teorías coherentes de relaciones internacionales de la globalización. Las razones de ello residen en la esencia de la globalización y en los fundamentos medulares de las teorías de relaciones internacionales convencionales. Estas razones, separadas y combinadas, nos llevan a concluir que cualquier intento de desarrollar teorías coherentes acerca de la globalización sigue siendo una tarea difícil.

\section{a) El problema de definir claramente a la globalización:} ¿cuál es el objeto de estudio?

Uno de los principales problemas de cualquier esfuerzo por teorizar sobre la globalización es el hecho de que se trata de un concepto vago e ilusorio. Esta confusión es de hecho una de las características del concepto, y es según Fiss y Hirsch lo que la vuelve, por un lado, uno de los conceptos más estudiados en el discurso académico contemporáneo y, por otro lado, uno de los más cues- 
tionados. ${ }^{19}$ En efecto, en la bibliografía de relaciones internacionales existente no existe una definición común aceptada de la globalización. Por ejemplo, se ha entendido a la globalización como acción a la distancia, compresión de tiempo-espacio; interdependencia creciente, un mundo pequeño y que se encoge, creciente conexión entre sociedades, desterritorialización de las relaciones internacionales y un conjunto de procesos que encarnan una transformación de la organización espacial de las relaciones y transacciones sociales. ${ }^{20}$

Claramente, esta confusión inherente plantea un gran desafío al desarrollo de teorías coherentes de relaciones internacionales sobre la globalización, principalmente porque, si no podemos ponernos de acuerdo sobre lo que es la globalización, ¿cómo podemos establecer las premisas y los supuestos centrales relativos a sus fuentes, efectos y trayectoria posible en una forma coherente, válida y plausible? En consecuencia, los debates teóricos acerca de la globalización tienden a quedarse en el plano ontológico, enfocándose principalmente en lo que se entiende por el término y sus alcances reales y potenciales, sea como fenómeno o como concatenación de procesos diversos y múltiples. ${ }^{21}$

\section{b) ¿¿Por qué es más difícil hacer teoría de la globalización en el ámbito político que en otros campos de las ciencias sociales?}

El uso primero y más común para las conceptualizaciones de "globalización" se ancló, casi exclusivamente, en el ámbito económico, por lo que la globalización con frecuencia se considera un proceso

19 Peer C. Fiss y Paul M. Hirsch, "The Discourse of Globalization: Framing and Sensemaking of an Emerging Concept", American Sociological Review, vol. 70, 2005, pp. 29-52.

${ }^{20}$ Véase Held y McGrew, "The Great Globalization Debate”, p. 3; Smith y Baylis, The Globalization of World Politics, p. 7; Jan Aart Scholte, "The Globalization of World Politics", en Smith y John Baylis, The Globalization of World Politics, 2001, pp. 13-32; Anthony Giddens, The Consequences of Modernity, Cambridge, Cambridge University Press, 1990; y Held et al., Global Transformations.

${ }^{21}$ Véase Hay, "Theories of Globalization", p. 3. 
y fenómeno económico que desafía el diseño del sistema económico mundial. Posteriormente, el concepto migró al campo de la sociología, principalmente con la concepción de la globalización como nociones desafiantes a la modernidad como proceso cultural o incluso hegemónico y que es aparente en nuestra vida diaria. ${ }^{22}$ Eventualmente, esas dos concepciones, la económica, que se enfoca en procesos que ocurren por encima y más allá del nivel estatal, y la sociológica, que subraya los procesos que ocurren debajo y más allá del nivel estatal, facilitan una visión política de la globalización como proceso que cuestiona, y que puede provocar, la desaparición del Estado territorial soberano.

Las teorías del campo de la economía y de la sociología parecen mejor preparadas para tratar con la globalización y conceptualizarla, una vez que logran trascender la carga de teorizar los efectos de la globalización en los Estados-nación y el sistema interestatal; por consiguiente, les resulta más fácil ajustarse a la condición en la que la vida social y política se ven cada vez menos restringidas por limitaciones territoriales. Esto es posible en economía, considerando que las teorías se mantienen en el nivel práctico, incluso matemático, y establecen las medidas para la economía global. ${ }^{23}$ En el campo de la sociología, también es posible "desterritorializar" las relaciones internacionales una vez que el foco se mueve a las interacciones organizacionales y culturales a varios niveles, con que se trascienden los marcos específicos y estrechos del Estado y del sistema estatal. ${ }^{24}$

22 Véase Philip G. Cerny, Rethinking World Politics: A Theory of Transnational Neopluralism, Oxford, Oxford University Press, 2010, p. 619; Hurrell, On Global Order, p. 192.

${ }^{23}$ Véase por ejemplo Nancy Brune y Geoffrey Garett, "The Globalization Rorschach Test: International Economic Integration, Inequality, and the Role of Government”, Annual Review of Political Science, vol. 8, 2005, pp. 402-412; Duane Swan, "The Effect of Globalization on Taxation, Institutions, and Control of the Macroeconomy", en David Held y Anthony McGrew (eds.), The Global Transformation Reader: An Introduction to the Globalization Debate, Londres Polity, 2003, pp. 403-419.

${ }^{24}$ Véase Jens Bartelson, "Is There a Global Society?”, International Political Sociology, vol. 3, núm. 1, 2009, p. 113; Ronald Robertson, "Differentiational Reductionism and the Missing Link in Albert's Approach to Globalization Theory", International Political Sociology, vol. 3, núm. 1, 2009, p. 122. 
Tanto para la economía como para la sociología, el Estado y sus aspectos distintivos no son necesariamente parte de las fundaciones mismas de los principales paradigmas disciplinarios, de aquí que sea más fácil tomar e incorporar nociones de globalización en sus programas de investigación. En ambas disciplinas, las unidades básicas de análisis no son los Estados, sino los individuos, las firmas y las sociedades: un despliegue de actores que ontológicamente están separados del Estado, de manera que pueden ser pensados con mayor facilidad dentro de contextos variables políticos, sociales, económicos y espaciales. Con todo, de hecho, ni la economía ni la sociología tienen tampoco una teoría separada, plena o coherente de la globalización.

Por el contrario, el caso de la globalización en el ámbito político, especialmente en lo que se refiere a las relaciones internacionales, es mucho más demandante a este respecto, pues involucra una de las disputas más prominentes en el área en lo que se refiere a la posibilidad de cambio en la arena internacional y además nos mueve a reconsiderar una de las premisas centrales del área, a saber, la "gran brecha" entre los ámbitos políticos internos y externos.

Una de las maneras comunes para intentar reconciliar el concepto de globalización y para salvar la "gran brecha" puede verse en el desarrollo del concepto de política global en tanto que incorpora una esfera política que puede distinguirse del estudio tradicional de la relaciones internacionales dado que destaca el papel de lo "global" como un lugar político. La política global en tanto que representa relaciones políticas que trascienden fronteras es así neutral o al menos independiente de la concepción de la "gran brecha". Aún así, el empleo del concepto de política global, en tanto que se distingue de las relaciones internacionales, podría crear otra gran brecha, esta vez no entre lo interno y lo internacional, sino más bien entre lo internacional y lo global. Intentos recientes de establecer aproximaciones de análisis para estudiar la política global, sobre todo según los conceptos de gobernanza global y de sociedad global civil, pueden reforzar esta nueva brecha entre lo global y lo internacional. ${ }^{25}$

${ }^{25}$ Véase Mor Mitrani, "Global Civil Society and International Society Compete or Complete?”, Alternatives, vol. 38, núm. 2, 2013, pp. 172-188. 


\section{c) Problemas lógicos en las tentativas para teorizar sobre la globalización}

Una tercera explicación de los obstáculos para formular teorías coherentes de relaciones internacionales sobre la globalización se relaciona con los problemas lógicos inherentes a la comprensión de su dinámica en términos de cambio y continuidad. En otras palabras, la globalización como resultado no puede explicarse meramente invocando la globalización como un proceso que lleva hacia el mismo resultado. ${ }^{26}$ De aquí que no podamos estar de acuerdo con que la globalización sea causa, efecto o ambos.

A decir verdad, existen múltiples procesos de globalización que interactúan desigualmente, de maneras específicas y contingentes; son también complejos y ambiguos, y son simultáneamente económicos, sociales, políticos y culturales. Así, conforme el lenguaje de la globalización parece sugerir una combinación de "todo" (de ser y de convertirse, de proceso y de resultado, de causa y de efecto), éste crea un acertijo lógico insalvable. Se hace crucial entonces descomponer y revelar los procesos subyacentes que el concepto de globalización asume e implica, bien que al mismo tiempo sea casi imposible lidiar simultáneamente con la complejidad, la ambigüedad y aun la incoherencia de los fenómenos y procesos involucrados. Por consiguiente podemos concluir que aunque el concepto de globalización puede crear la impresión de una posibilidad de una explicación general, a decir verdad, acaso, no pueda por sí misma explicar nada. De ahí las dificultades de teorizar sobre ella. ${ }^{27}$

Como con la definición de globalización en sí misma, no hay consenso en la discusión con respecto a las fuerzas y procesos que impulsan la globalización. En primer lugar, atajando por la brecha paradigmática e ideológica de idealismo y materialismo,

${ }^{26}$ Véase Justin Rosenberg, "The Problem of Globalization Theory”, en Held y McGrew, The Global Transformation Reader, pp. 92-7; Luke Martell, "The Third Wave in Globalization Theory”, International Studies Review, vol. 9, núm. 2, 2007, p. 182.

27 Colin Hay y David Marsh, "Introduction: Demystifying Globalization”, en Colin Hay y David Marsh (eds.), Demystifying Globalization, Handmills, Macmillan Press, 2000, p. 10. 
la globalización puede entenderse a la vez como resultado de actores individuales (orientada al agente) o como la reflexión y proyección de fuerzas estructurales. Los argumentos individualistas se refieren a la globalización como un resultado de decisiones hechas por actores sociales ("agentes"), como ciudadanos, funcionarios, políticos, empresarios, miembros de la sociedad civil e intelectuales. Por el contrario, las perspectivas estructuralistas contemplan la globalización como resultado de profundas fuerzas estructurales como el capitalismo global, el surgimiento, evolución y probable declive del sistema del Estado, y la convergencia y superposición del nacionalismo, el regionalismo y el internacionalismo. $^{28}$

Con todo, cuando se intenta explicar por qué el desafío de analizar la globalización, sus fuentes y sus efectos posibles torna la teorización sobre la globalización en un reto casi imposible en general, y en las relaciones internacionales en particular, arribamos naturalmente a un debate importante sobre cambio y continuidad en el sistema internacional. La sola idea de globalización y los tanteos concomitantes para teorizarla están anclados en el concepto básico de cambio. Como se anotó arriba, todas las definiciones y conceptualizaciones de globalización asumen en su esencia cambios cuantitativos y cualitativos en aspectos heterogéneos de procesos complejos. No sorprende entonces que la principal crítica de los expertos globalistas contra las teorías convencionales de relaciones internacionales en el contexto de la globalización sea que estas teorías ya no son adecuadas para explicar y entender la política mundial. Como quiera que sea, el rechazo de las teorías de relaciones internacionales es básicamente histórico y reposa sobre la premisa de que los procesos de globalización habían vuelto del todo obsoletos a los modelos estadocéntricos del orden westphaliano con tal de dar sentido a los asuntos mundiales contemporáneos.

Críticas parecidas pueden encontrarse en las vertientes críticas de la teoría de la globalización que declaran que la teoría de las relaciones internacionales es incapaz de entender el ámbito

${ }^{28}$ Véase Scholte, Globalization: A Critical Introduction, p. 21. 
global, a la vez que la globalización plantea desafíos ontológicos y epistemológicos con los que las teorías de las relaciones internacionales no pueden reconciliarse ${ }^{29}$ Estas aproximaciones críticas son diferentes de nuestra afirmación de que mientras que los Estados sean capaces de mantener su rol autoritario en la política mundial, y mientras que las relaciones interestatales dominen las transacciones políticas, deberíamos desarrollar teorías que puedan explicar y entender la globalización desde una perspectiva de las relaciones internacionales, y no al contrario. Hacemos hincapié entonces en una aproximación política que valore la globalización desde el punto de vista de las teorías de relaciones internacionales. En otras palabras, y yuxtapuesto a los enfoques críticos que apuntan a déficits teóricos parecidos en relaciones internacionales, sostenemos que las dimensiones globales e internacionales (es decir, interestatales) de la política global no son necesariamente contradictorias ni mutuamente excluyentes.

Pese a la supuesta vaguedad de la teoría de la globalización, podemos edificar a partir de las tentativas heroicas ya mencionadas para moverse de lo que se ha denominado "territorialismo metodológico", ${ }^{30}$ lo cual, puede decirse, está acoplado a la definición misma de relaciones "inter-nacionales", por efecto de discutir la relevancia de la gobernanza global y de la gobernanza regional como instrumentos útiles para teorizar sobre las relaciones internacionales a lo largo de distintas áreas temáticas, que van de las crisis económicas a los retos ambientales. ${ }^{31}$

29 Véase, por ejemplo, Jens Bartelson, "Three Concepts of Globalization", International Sociology, vol. 15, núm. 2, 2000, pp. 180-196; Jens Bartelson, "The Social Construction of Globality", International Political Sociology, vol. 4, núm. 3, 2010, pp. 219-35; Nisha Sha, "The Territorial Trap of the Territorial Trap: Global Transformation and the Problem of the State's Two Territories", International Political Sociology, 6, 1, 2012, pp. 57-76

30 Citado Rosenberg, "The Problem of Globalization Theory”, p. 94.

31 Véase Thomas G. Weiss y Rordern Wilkinson, "Global Governance to the Rescue: Saving International Relations?”, Global Governance, 20, 1, 2014, pp. 19-36; y Tom Pegram y Michele Acuto, "Global Governance in the Interregnum", Millenium, 2015. 
d) El prejuicio territorial de la disciplina de las relaciones internacionales y el problema de la "gran brecha"

La explicación de arriba conduce directamente al tema del prejuicio territorial, del foco estadocéntrico y de la relevancia persistente del paradigma realista en relaciones internacionales. Según arguye sucintamente Hay, el debate en teoría de relaciones internacionales sobre la globalización es, en efecto, una disputa ontológica entre el estadocentrismo y el no estadocentrismo. ${ }^{32}$

Como argumenta Cerny, la innovación central de la globalización, como concepto y como discurso, es el hecho de que desafía las categorizaciones tradicionales de niveles de análisis que habituamos para estudiar, enseñar y dar sentido del mundo empírico de las relaciones internacionales. La distinción entre los ámbitos internacional y doméstico había establecido el núcleo de la disciplina y práctica de las relaciones internacionales desde Westphalia, lo que creó una "gran brecha" artificial que formó nuestros propios prejuicios para representar el mundo y para explicarlo. ${ }^{33} \mathrm{De}$ esta manera, la globalización desafía y subvierte esta "gran brecha" entre la política interna y la política internacional, así como al prejuicio territorial de la disciplina de las relaciones internacionales.

Más aún, este "territorialismo metodológico" de prejuicio estadocéntrico de las relaciones internacionales explica también las dificultades de la disciplina de relaciones internacionales para ponerse de acuerdo para lidiar con conceptos y realidades como "globalización", "sociedad global (o mundial)" y "cosmopolitanismo". Muchas teorías de relaciones internacionales asumen todavía que nuestra área de investigación se limita sobre todo a las interacciones entre sociedades políticas acotadas, en un contexto general definido por la falta de autoridad centralizada ("anarquía"). Esto puede explicar asimismo por qué los especialistas en relaciones internacionales pueden desarrollar conceptos tales como "interdependencia compleja" o inclusive argüir sobre "gobernanza global",

${ }^{32}$ Hay, "Theories of Globalization", pp. 4-6.

${ }^{33}$ Véase Cerny, Rethinking World Politics, pp. 620-621; y Clark, Globalization and International Relations Theory. 
hasta el extremo de que su territorialismo metodológico persiste aún, si bien son reacios en términos teóricos a aceptar el concepto más amplio de globalización, trascendiendo la lógica del Estado y del sistema estatal mismo.

El prejuicio territorial y la concepción de la "gran brecha" también conducen al supuesto dicotomista de suma cero sobre las relaciones entre globalización, el Estado y el sistema interestatal. A nuestro parecer, tanto globalistas como escépticos se equivocan en sus estimaciones contradictorias y extremas sobre el impacto de la globalización sobre el futuro del Estado-nación y el sistema estatal, especialmente cuando se lo presenta como una concepción dicotomista. Desde nuestro punto de vista, los Estados y los procesos de la globalización se constituyen unos a otros. Bajo esta premisa, los enfoques constructivistas perciben la globalización como una "interpretación particular de una realidad social que está siendo interpretada y reinterpretada por agentes sociales", ${ }^{34}$ y, con ello, como el resultado tanto como el generador de cambios sociales entre agentes y estructuras políticos. El impacto de la globalización sobre la política mundial se forma así por sus efectos constitutivos sobre normas y prácticas de varios agentes, y viceversa. A este respecto, la globalización no es una fuerza que cambie a los Estados a priori, sino que incluye procesos que los Estados (en tanto que agentes) "hacen" y (re)construyen. Dado que los agentes políticos no son entidades ni pasivas ni estáticas, sino que más bien son activos y responden a las estructuras, los cambios contemporáneos en las relaciones políticas no pueden entenderse solamente como los resultados de la globalización, sino como manifestaciones de las respuestas de los Estados a ella.

Muchos especialistas en globalización asumen que las fuerzas de la misma harán del Estado-nación un actor irrelevante en la política mundial; de aquí que entre más se difunda la globalización, menos funcionales se volverán los Estados (por ello, hay aquí una perspectiva de suma cero). Por el contrario, los escépticos mi-

${ }^{34}$ Véase Thomas Risse, "Social Constructivism Meets Globalization", en David Held y Anthony McGrew, Globalization Theory: Approaches and Controversies, Cambridge, Polity, 2007, p. 129. 
ran las fuerzas de la globalización como el reflejo de la distribución subyacente del poder y de la superioridad de los Estados; en otros palabras, entre más poderosos sean los Estados, menos relevantes o limitadas son las fuerzas de la globalización (de aquí que, nuevamente, enfrentemos una perspectiva de suma cero). Este debate es entonces redundante puesto que gira en torno a una hipotética "pregunta de sí o no", en vez de enfocarse en las gradaciones posibles; por ejemplo, los intentos de las instituciones internacionales, por medio de la cooperación interestatal, para lidiar exitosamente con la crisis financiera luego de 2008, o la dispersión gradual del poder desde Estados Unidos en dirección de grandes potencias emergentes y actores no estatales. Además, el debate entre globalistas y escépticos es también estéril, pues trata con un resultado final que nadie puede ubicar con certeza en el continuo de tiempo y espacio, a la vez que presupone que estos elusivos procesos que denominamos "globalización" son predeterminados, lineales y claramente demarcados. A este respecto, la mayor parte de la bibliografía sobre las dimensiones políticas de la globalización tiende a enfocarse, erróneamente, en la viabilidad del Estado-nación en una era global, y a delinear las trayectorias supuestamente predeterminadas de la globalización en la dirección del nirvana humano, el cosmopolitanismo, la gobernanza global y, al final, el gobierno mundial.

De hecho, sugerimos que la respuesta para explicar y entender la globalización reposa en alguna parte del medio, dando prioridad al papel persistente del Estado-nación en el marco más amplio del ámbito internacional en que se lidia con la globalización. Desde este punto de vista, los debates relevantes deberían girar no sólo en torno a la pura viabilidad del Estado, sino además en torno a las alteraciones en su carácter, a saber, el concepto cambiante y el contenido de la soberanía del Estado, la autoridad y la legitimidad. Así, este enfoque de terreno medio nos ayuda a responder apropiadamente a preguntas como: ¿Qué ha cambiado en la naturaleza del Estado y en sus capacidades para cumplir funciones? ¿Cómo es que los Estados manejan los procesos de globalización y sus efectos? Es asimismo la salida lógica, si no de sentido común, 
atender las tres cuestiones planteadas en este artículo en una dirección positiva (normativamente hablando).

La abarrotada agenda de la doble difusión del poder y de las crisis múltiples de la globalización en varias áreas temáticas hace del llamado a teorizar en las relaciones internacionales aún más relevante. Pero aquí hay una paradoja. La compartimentación actual de la globalización en sus diferentes facetas y dimensiones produce un sentido de premura para teorizar sobre ella; aún así, al mismo tiempo, vuelve muy difícil a este proyecto teórico, si no imposible.

Al fracasar en el razonamiento sobre la globalización, o al dar por sentadas las realidades de la globalización, las teorías de relaciones internacionales pueden crear un vacío teórico que las hará inadecuadas para explicar y entender la estructura y las fuerzas que animan la arena global contemporánea. De esta manera, incluso a costa de la incoherencia, la confusión y los enfoques discutibles, las teorías de relaciones internacionales deberían esmerarse en actualizar y adaptar sus marcos teóricos e instrumentales, adecuándolos a una realidad cambiante en que las relaciones internacionales tengan lugar en un mundo globalizado, definidor y definido por procesos de globalización, por efecto de mecanismos de gobernanza global y regional.

Esto podría ser la base para un plan de investigación más relevante de las relaciones internacionales contemporáneas en un mundo globalizado. Esta agenda de investigación debiera enfocarse en el papel que los Estados aún tienen en la política mundial, si bien por mecanismos y estructuras diferentes y transformados. Es tiempo de arrojar luz no sólo en el mero desarrollo de estas estructuras regionales y de gobernanza, sino también en las interacciones interestatales que construyen estos mecanismos y que, así, dotan de nuevo significado al concepto de "relaciones internacionales". Esto permitirá desviar la atención de la necesidad de entender el mayor y debatido concepto de globalización, en favor de la manifestación específica de las relaciones internacionales y el comportamiento internacional en un mundo globalizado y en cómo se lo gestiona y gobierna sin un gobierna mundial formal. 
Por ejemplo, en el ámbito de la seguridad, con el desarrollo de mecanismos de gobernanza de seguridad regional, estamos ante una creciente interacción interestatal entre las organizaciones y dentro de ellas, en las regiones y a lo largo de ellas y entre los esquemas de gobernanza global y regional. Presenciamos un número cada vez mayor de operaciones de paz conjuntas, entre la onU y la Unión Africana (en Darfur), entre la oтAN y la Unión Europea (en los Balcanes, en Libia), entre la otan y la onu (en la ex-Yugoslavia, Afganistán, Iraq, Libia, junto con la Unión Africana). Las actividades del contraterrorismo muestran también diversas configuraciones de los actores globales, regionales y nacionales. En todos estos casos, los Estados y sus relaciones internacionales desempeñan un papel dominante que tenemos que entender y explicar en términos de motivos políticos, poder y prácticas. Por una parte, las grandes potencias como Estados Unidos, China, Rusia y la Unión Europea, que son los proveedores primordiales de seguridad en términos unilaterales y bilaterales, emplean cada vez más estos variados escenarios multilaterales para promover sus propios objetivos de seguridad, a menores costos. Por otra parte, simultáneamente, los Estados regionales o pivote también utilizan las mismas organizaciones para incrementar su voz e introducir su gestión de seguridad regional, a fin de mitigar el abrumador poder material de las grandes potencias extra-regionales y para fortalecerse en una gobernanza de seguridad regional más efectiva cuando las potencias extra-regionales no puedan intervenir o aportar los bienes colectivos necesarios.

\section{Conclusiones}

Sin duda, la globalización es uno de los conceptos y realidades más comunes y relevantes en el estudio contemporáneo de las relaciones internacionales. Sin embargo, mientras que muchos académicos e investigadores exploran y escudriñan el concepto y las realidades de la globalización y sus efectos en la esfera política, específicamente en el sistema internacional, no se han desarrollado todavía teorías coherentes de relaciones internacionales acerca de la 
globalización, o no han logrado encontrar su lugar dentro de las corrientes teóricas convencionales de relaciones internacionales. Esto nos resulta bastante peculiar dado que si en efecto la globalización puede considerarse una idea y concepto principal en los órdenes sociales contemporáneos, uno podría esperar cada vez más intentos de desarrollar teorías capaces de evaluar las realidades actuales y de ofrecer lentes adecuadas para entenderlas y explicarlas.

Lo que está en juego analíticamente es evaluar si las principales afirmaciones de las teorías de relaciones internacionales son aún compatibles con el concepto y las realidades de la globalización. En este sentido, expandimos nuestra discusión al ámbito de la política global, y hemos desarrollado un contexto que da prioridad al papel persistente de los Estados con el fin de evaluar los intentos previos de varios especialistas en relaciones internacionales para desarrollar teorías en ese campo sobre la globalización, incluso si no han sido completamente exitosas.

Desde nuestro punto de vista, el principal puente entre las teorías de relaciones internacionales y la globalización es la política manifestada en la acción de los Estados en la sociedad internacional. La noción creciente de un orden post-westfaliano parece pasar por alto el hecho de que como los Estados desempeñan un papel decisivo y prominente en la política mundial, si bien ese papel se ha transformado y adaptado, cualquier conceptualización de marcos de un "antes" y un "después" es engañoso. El orden no se convierte en un post-orden de la noche a la mañana, y la tendencia a presentarlo así ignora los procesos graduales mediante los cuales los agentes responden y se adaptan a los cambios más que simplemente ser transformados por esos cambios. El principal argumento en este respecto es que sería un error tratar a la globalización como una fuerza independiente en sí misma. La globalización por sí sola no puede cambiar al Estado o a las relaciones inter-estatales, ni volver post-westfalianos al sistema o al orden internacional. Los Estados siguen siendo los agentes principales en la política mundial y como tales responden a la globalización y se adaptan, así como sus patrones de relaciones y prácticas, con el fin de ser relevantes y adecuados para las nuevas realidades de la globalización. 
Dado el escaso inventario de teorías de la globalización que existe en relaciones internacionales, en este artículo señalamos varias dificultades inherentes que existen en la formulación de teorías coherentes de relaciones internacionales sobre la globalización y subrayamos la confusión general del concepto y el problema de reconciliarlo con las premisas centrales de las teorías de relaciones internacionales. En el artículo, identificamos dos fases secuenciales en la teorización sobre la globalización. La primera etapa se enfocó en los intentos de definir y explicar la globalización como un todo, con que se da sustancia a la idea de política global. Una fase posterior, que se desarrolló desde la crisis económica global de 2008, se concentró más en las consecuencias posibles de la globalización al describir y subrayar las áreas temáticas específicas que han sido moldeadas por la interacción de la globalización con actores estatales y no estatales; esto ha llevado a actores estatales, instituciones internacionales y actores no estatales por igual a practicar la gobernanza global.

A pesar de la extensa bibliografía sobre ambas fases, hemos puesto el acento en la necesidad de desarrollar herramientas teóricas afianzadas en la teoría de relaciones internacionales, para explicar cómo la globalización ha afectado a las relaciones internacionales, es decir, las relaciones inter-estatales per se. Por tanto, debemos cambiar la secuencia lógica de nuestras preguntas, fijándonos menos en si y en cómo la globalización afecta al Estado o a las relaciones inter-nacionales, y más en cómo los Estados responden a las dimensiones y realidades de la globalización. Así pues, no obstante los problemas, obstáculos e impedimentos para desarrollar teorías coherentes de relaciones internacionales, nuestra misión no es una causa completamente perdida. Eso se puede hacer, o intentar hacerse, si se explica y comprende lo que significan los diversos procesos de globalización desagregándolos en dimensiones y procesos más concretos, por ejemplo mediante los mecanismos políticos y formales de la gobernanza regional y global diseñados para proveer bienes colectivos y enfrentar los desafíos de la globalización. La necesidad analítica reside no sólo en describir y exponer las manifestaciones globales de áreas temáticas específicas contemporáneas, como la pobreza, desigualdad, 
democracia, derechos humanos, medio ambiente, proliferación nuclear y otras por el estilo. Más bien, debemos teorizar acerca de las condiciones bajo las cuales los Estados y otros actores colectivamente enfrentan y lidian con esos asuntos globales de manera coherente; para ello hay que ocuparnos de las prácticas comunes de las relaciones internacionales, con que se explique la política mundial con mecanismos de gobernanza global y regional.

Se podría decir que es una conclusión obvia o, peor aún, que este ejercicio intelectual no contribuye realmente a las discusiones teóricas que aspiran a conceptualizar y entender más los procesos de globalización y gobernanza global en general, y en el contexto de las relaciones internacionales en particular. Sin embargo, debemos advertir las responsabilidades, propósitos y limitaciones que tenemos como estudiosos de las relaciones internacionales. La brecha entre el reconocimiento común de que la globalización es una realidad apremiante y nuestra capacidad de elaborar teorías coherentes sobre la misma merece plena atención. Nos apoyamos en paradigmas, teorías y programas de investigación para dar sentido de manera sistemática a la complejidad de nuestro mundo. Globalización es en efecto el concepto que usamos para indicar que algunos de los órdenes y principios mundiales previos a los que estábamos habituados han sufrido profundas transformaciones, que por lo común se caracterizan por su incoherencia y confusión. De hecho, no sorprende entonces que enfrentemos grandes dificultades para articular y formular las transformaciones dentro de los esquemas teóricos coherentes que todos estamos entrenados a desarrollar y formular. Por consiguiente, proponemos una agenda de investigación más estructurada que desagregue el concepto de globalización en sus diferentes dimensiones, aspectos y temas. Ya observamos esa tendencia en la discusión acerca de las diferentes dimensiones de la gobernanza global y regional, en los ámbitos de la seguridad y la economía (entre otros).

Sin embargo, enfrentamos una paradoja de doble filo. Por un lado, el concepto de globalización parece destacar las redundancias y limitaciones contemporáneas de las teorías de relaciones internacionales. Pero, por otro lado, sabemos que las relaciones internacionales per se, a saber las prácticas e instituciones de las 
interacciones interestatales, están lejos de ser redundantes u obsoletas en nuestros días. Por lo tanto, el hecho de que los teóricos de las relaciones internacionales encuentren difícil adaptar y aplicar las teorías al concepto y las realidades de la globalización no significa que en la práctica las relaciones internacionales entre Estados, que son todavía el objeto central de nuestro campo, no se estén adaptando y transformando ellas mismas, a la luz y en respuesta a esos mismos procesos de globalización.

Por lo tanto, las realidades cambiantes de las relaciones internacionales una vez más toman precedencia sobre nuestros esfuerzos teóricos de dar sentido al mundo. Así, proponemos una agenda de investigación que se enfoca en los fenómenos empíricos de cómo los Estados, en la práctica, se adaptan y ajustan a estos procesos dinámicos, al tiempo que mantenemos lo "internacional” junto con lo "global", en lugar de reemplazar el primero con el segundo. En este valiente mundo nuevo de globalización, creemos que aún hay espacio para teorías coherentes de relaciones internacionales, quizá no totales, pero igualmente válidas y útiles para dar sentido a la interacción entre los ámbitos internacional y global, donde los Estados todavía juegan un papel primordial como agentes de cambio y transformación.

Traducción de Gabriel Ramos

\section{BiBLIOGRAFÍA}

Bartelson, Jens, "Three Concepts of Globalization”, International Sociology, vol. 15, núm. 2, 2000, pp. 180-196.

, "Is There a Global Society?", International Political Sociology, vol. 3, núm. 1, 2009, pp. 112-115.

, "The Social Construction of Globality", International Political Sociology, vol. 4, núm. 3, 2010, pp. 219-235.

Beck, Ulrich, The Reinvention of Politics: Rethinking Modernity in the Global Social Order, Cambridge, Polity Press, 1997.

Brune, Nancy y Geoffrey Garett, “The Globalization Rorschach Test: 
International Economic Integration, Inequality, and the Role of Government”, Annual Review of Political Science, vol. 8, 2005, pp. 402-412.

Buzan, Barry, From International to World Society? English School Theory and the Social Structure of Globalization, Cambridge, Cambridge University Press, 2004.

Cerny, Philip G., Rethinking World Politics: A Theory of Transnational Neopluralism, Oxford, Oxford University Press, 2010.

Clark, Ian, "Beyond the Great Divide: Globalization and the Theory of International Relations", Review of International Studies, vol. 24, núm. 4, 1998.

- Globalization and International Relations Theory, Oxford, Oxford University Press, 1999, p. 5.

—, "Globalization and the Post-Cold War Order", en Steve Smith y John Baylis (eds.), The Globalization of World Politics: An Introduction to International Relations, Oxford, Oxford University Press, 2001, pp. 639-646.

Drezner, Daniel W., "The Irony of Global Economic Governance: The System Worked”, Council on Foreign Relations International Institutions and Global Governance Program, Working Paper, 2012.

Falk, Richard, "A Radical World Order Challenge: Addressing Global Climate Change and the Threat of Nuclear Weapons", Globalizations, vol. 7, núms. 1-2, 2010, pp. 137-155.

Fiss, Peer C. y Paul M. Hirsch, "The Discourse of Globalization: Framing and Sensemaking of an Emerging Concept", American Sociological Review, vol. 70, 2005, pp. 29-52.

Giddens, Anthony, The Consequences of Modernity, Cambridge, Cambridge University Press, 1990.

Gills, Barry K., "The Return of Crisis in the Era of Globalization: One Crisis or Many?”, Globalizations, vol. 7, núms. 1-2, 2010, pp. 3-8.

Hay, Colin, “Theories of Globalization”, en Tim Dunne, M. Kurki y Steven Smith (eds.), International Relations Theories: Discipline and Diversity, Oxford, Oxford University Press, 2007.

Held, David y Anthony McGrew, "The Great Globalization Debate: An Introduction", en David Held y Anthony McGrew (eds.), The Global Transformation Reader: An Introduction to the Globalization Debate, Londres, Polity, 2003, pp. 1-50.

Hay, Colin y David Marsh, "Introduction: Demystifying Globalization", en 
Colin Hay y David Marsh (eds.), Demystifying Globalization, Handmills, Macmillan Press, 2000.

Hurrell, Andrew, On Global Order: Power, Values, and the Constitution of International Society, Oxford, Oxford University Press, 2007.

Kacowicz, Arie M., Globalization and the Distribution of Wealth: The Latin American Experience, 1982-2008, Cambridge University Press, 2013.

Kacowicz, Arie M. y Galia Press-Bar-Nathan, "Regional Security Governance", en Tanja Boerjel y Thomas Risse (eds.), Oxford Handbook of Comparative Regionalism, Oxford, Oxford University Press, 2016.

Martell, Luke, "The Third Wave in Globalization Theory", International Studies Review, vol. 9, núm. 2, 2007.

Mitrani, Mor, "Global Civil Society and International Society Compete or Complete?”, Alternatives, vol. 38, núm. 2, 2013, pp. 172-188.

Mittelman, James H., "Crisis and Global Governance: Money, Discourses, and Institutions", Globalizations, vol. 7, núms. 1-2, 2010, pp. 157-172.

Naim, Moises, The End of Power: From Boardrooms to Battlefields and Churches to States, Why Being in Charge Isn't What Is Used to Be, Nueva York, Basic Books, 2013.

Pegram, Tom y Michele Acuto, "Global Governance in the Interregnum", Millenium, 2015.

Risse, Thomas, "Social Constructivism Meets Globalization", en David Held y Anthony McGrew, Globalization Theory: Approaches and Controversies, Cambridge, Polity, 2007.

Robertson, Ronald, "Differentiational Reductionism and the Missing Link in Albert's Approach to Globalization Theory", International Political Sociology, vol. 3, núm. 1, 2009, p. 122.

Rosenau, James N. y E. O. Czempiel (eds.), Governance without Government: Order and Change in World Politics, Cambridge, Cambridge University Press, 1992.

Rosenau, James N., "Toward and Ontology for Global Governance”, en Martin Hewson y Timothy J. Sinclair (eds.), Approaches to Global Governance Theory, Nueva York, suny Press, 1999.

, "Change, Complexity and Governance in Globalizing Space", en J. Pierre (ed.), Debating Governance: Authority, Steering and Democracy, Oxford, Oxford University Press, 2000.

-, "Turbulence in World Politics: A Theory of Change and Continuity”, en Richard W. Mansbach y Edward Rhodes (eds.), Global 
Politics in a Changing World: A Reader, Boston, Houghtin Miffin Co., 2003, pp. 21-22.

, "Governance in the $21^{\text {st }}$ Century", en Rorden Wilkinson (ed.), The Global Governance Reader, Londres, Routledge, 2005, pp. 45-46.

Rosenberg, Justin, "The Problem of Globalization Theory", en Held y McGrew, The Global Transformation Reader, pp. 92-97.

Scholte, Jan Aart, "The Globalization of World Politics", en Smith y John Baylis, The Globalization of World Politics, 2001, pp. 13-32.

-, Globalization: A Critical Introduction, 2a ed., Nueva York, PalgraveMacmillan, 2005, p. 121.

Sha, Nisha, "The Territorial Trap of the Territorial Trap: Global Transformation and the Problem of the State's Two Territories", International Political Sociology, 6, 1, 2012, pp. 57-76

Swan, Duane, "The Effect of Globalization on Taxation, Institutions, and Control of the Macroeconomy", en David Held y Anthony McGrew (eds.), The Global Transformation Reader: An Introduction to the Globalization Debate, Londres Polity, 2003, pp. 403-419.

Weiss, Thomas G. y Rordern Wilkinson, "Global Governance to the Rescue: Saving International Relations?”, Global Governance, vol. 20, núm. 1, 2014, pp. 19-36. 